International Journal of Applied Dental Sciences 2020; 6(4): 08-13

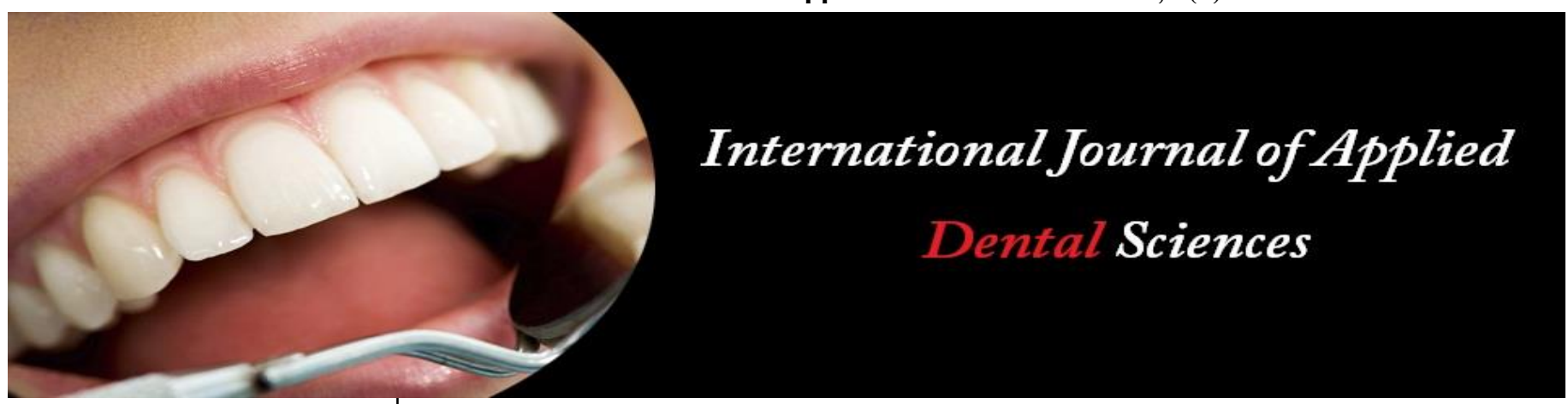

ISSN Print: 2394-7489

ISSN Online: 2394-7497

IJADS 2020; 6(4): 08-13

(C) 2020 IJADS

www.oraljournal.com

Received: 27-07-2020

Accepted: 10-09-2020

Nathalia Decarli Presotto

Postgraduate Student, Dentistry

Course, School of Health and

Life Sciences, Pontifical Catholic

University of Rio Grande do Sul,

Porto Alegre, Brazil

Fernanda Cásseres Teixeira

Postgraduate Student, Dentistry

Course, School of Health and

Life Sciences, Pontifical Catholic

University of Rio Grande do Sul

Porto Alegre, Brazil

Aline Rose Cantarelli Morosolli Adjunct Professor, Discipline of Radiology, Dentistry Course,

School of Health and Life Sciences, Pontifical Catholic

University of Rio Grande do Sul,

Porto Alegre, Brazil

Maria Ivete Bolzan Rockenbach Adjunct Professor, Discipline of Radiology, Dentistry Course, School of Health and Life Sciences, Pontifical Catholic University of Rio Grande do Sul, Porto Alegre, Brazil

Corresponding Author:

Maria Ivete Bolzan Rockenbach Adjunct Professor, Discipline of Radiology - Dentistry Course -

School of Health and Life

Sciences, Pontifical Catholic

University of Rio Grande do Sul-

Porto Alegre, Brazil

\section{Errors in the periapical radiographic technique}

\section{Nathalia Decarli Presotto, Fernanda Cásseres Teixeira, Aline Rose Cantarelli Morosolli and Maria Ivete Bolzan Rockenbach}

DOI: $\underline{\text { https://doi.org/10.22271/oral.2020.v6.i4a.1039s }}$

\section{Abstract}

Purpose: The purpose of this study was to analyze the implementation of the periapical radiographic technique, when performed by dental students, whilst using a digital sensor and a phosphor imaging plate as image receptors, instead of radiographic film.

Materials and Method: Thirty (30) dentistry students in their fourth year were randomly selected for the execution of a full mouth periapical examination of the mandible and the maxilla, when performed on a mannequin.

Result: There was a greater frequency of radiographic technique errors with the use of the sensor than with the phosphor plate. Among the errors observed, the highest frequency was the absence of a safety margin for the sensor $(35.7 \%$ ), while for the phosphor plate, it was an overlapping of the proximal faces $(23.1 \%)$. When using both of the systems, the region of the lower right premolars presented the highest percentage of errors. With the use of the phosphor plate, $7.61 \%$ of the images were repeated, with a maximum of 2 replicates in 4 radiographs. While with the sensor, $38.57 \%$ needed to be repeated and 4 of these were repeated 9 times. McNemar test revealed statistically significant differences for the errors of a poor centering, an excessive safety margin and a non-parallel safety margin, between the images taken with the phosphor plate and with the sensor.

Conclusion: The greatest number of errors and repetitions occurred with the use of the digital sensor. This has emphasized the need for a greater training of the dentistry students with these systems, in order to reduce the radiation exposures and to protect the patients.

Keywords: Diagnostic errors, diagnostic imaging, digital dental radiography, learning

\section{Introduction}

The process of obtaining radiographic images is in transition. This is because the conventional radiographic system, which uses radiographic films as image receivers, is gradually being replaced by digital systems that have become the receivers of images, phosphor plates, or sensors. The introduction of these digital systems has represented a major advance in the diagnostic area, due to the several possibilities of manipulating the images, together with the ease of obtaining, storing and transmitting the radiography.

Radiographs play a fundamental role in the analysis and in the evaluation of the teeth and the jaws ${ }^{[1]}$. A radiographic examination has become an indispensable component of diagnostic imaging, since it provides information that is necessary to indicate, or to suggest, the presence or the absence of a pathology, as well as to plan and monitor the treatment. In dentistry, the most frequently used radiography is for the periapical, which is performed by the bisecting technique.

Thus, when considering the execution of the radiographic technique and the possibility of errors that occur during the exposure of X-ray image (XR) receptors, it is important to identify those that occur more frequently. This is in order to draw targets to decrease or to prevent these errors from occurring, in order to obtain an adequate radiograph for the diagnosis. This is also most especially for the protection of the patients against the ionizing radiation.

The aim of this study was to perform an analysis of the periapical radiographic technique, when performed by dental students, whilst using a digital sensor and a phosphor plate as image receptors, instead of radiographic film. The errors that occurred in obtaining the periapical radiography when using the two digital systems were identified, quantified and compared. 


\section{Materials and methods}

This study was approved by the Research Ethics Committee of the Pontifical Catholic University of Rio Grande do Sul (PUCRS), Brazil (Protocol \# 2.058.595).

For the execution of the periapical radiographic technique, thirty (30) students of dentistry in their fourth year and who had already studied the discipline of Dental Radiology III were randomly selected. The periapical radiographs were performed on the same mannequin, by using positioners that were suitable for the techniques, when using the sensor or the phosphor plate. For the acquisition of the digital periapical images, the Fona CDR Elite Sensor, powered by the Schick Digital System (Sirona Dental, Inc., Long Island City, New York, USA), was used as an image receptor. This sensor has CMOS-APS (complementary metal oxide semiconductor) technology. The sensor was of size \#2, which has an external area of $30 \times 43 \mathrm{~mm}^{2}$ and an active area of $26 \times 36.5 \mathrm{~mm}^{2}$, with a resolution of $27 \mathrm{ppl} / \mathrm{mm}^{2}$ (line pairs per square millimeter). The processing and the analyses of the images were performed when using the software that was specific to this digital system. A photo-sensitive phosphor plate (size \#2) from the DenOptix ${ }^{\circledR}$ Digital System (Gendex, Des Plaines, IL, USA) was also used as an image receptor, in order to obtain the digital radiographic images. The processing of these radiographic images was performed in the DenOptix ${ }^{\circledR}$ Digital System (Gendex, Des Plaines, IL, USA), by means of a drum-type laser scanner, when using the VixWin 2000 program (Gendex, Des Plaines, IL, USA).

In order to determine the exposure time, the electrical factors of the X-ray apparatus, the area to be X-rayed and the type of image receptor used (the sensor or the phosphor plate), these were all carefully considered.

The X-ray apparatus that was used to obtain the periapical radiographs was the Sommo (Gnatus, Ribeirão Preto, SP, Brazil), with an electric regime of $70 \mathrm{kVp}, 7 \mathrm{~mA}$ and with a $2.5 \mathrm{~mm}$ aluminum filter. For each student, 28 digital radiographs were requested, fourteen (14) exposures when using the sensor \#2 and fourteen (14) exposures when using the \#2 phosphor plate. They were able to repeat the radiographs, if they deemed it was necessary. The exposures included the molars, the premolars, the canines and the incisor regions, in both the upper and the lower jaws. The students were identified with numbers from 1 to 30 and the images were organized in digital files for a later analysis.

After each student had performed the radiographs, they received a questionnaire, with questions that were related to the two digital systems that they had used. The answers were analyzed and they were included in this study, in order to reach a conclusion about the advantages and the difficulties of the two systems, together with which system the students preferred to use.

The digital radiographs were evaluated by a trained and calibrated observer. They were analyzed individually, in a controlled lighting environment, by means of a properly calibrated visualization monitor, with physical characteristics, according to the visualization needs. The observer's calibration was initially conducted with the analysis of a series of selected images, together with the supervisor that was responsible for the research. In a second step, the evaluator analyzed another group of images at two different times, with a one-week interval between the observations. In order to evaluate the intra-rater reliability, the Kappa test was performed. The digital radiographs were classified according to the identified errors that were related to the radiographic technique, such as: a) an incorrect vertical angulation: shortened or elongated images; b) an incorrect horizontal angulation: beam of the X-ray not parallel to the proximal surfaces of the teeth; c) an incorrect incidence point: a cone cutting image; and d) an incorrect positioning of the receptor, the sensor or the phosphor plate; (to give examples: an excessive or the absence of a safety margin; the safety margin not being parallel to the incisal or the occlusal surface; an incorrect long axis of the receptor; a poor receptor centering). The statistical analysis of this study was performed by using descriptive statistics, when considering the frequency of the occurrence of the radiographic technique errors, together with a comparison between the groups. The McNemar test was used, in order to compare the digital systems with the various occurrences of the errors. The results were considered to be statistically significant at 0.05 .

\section{Results}

A total of 840 periapical digital radiographs were evaluated. They were performed in all of the regions of the maxilla and mandible. Of these, 420 radiographs were performed with the sensor and 420 with the phosphor plate.

The intra-rater reliability was evaluated by using the Kappa test. The values that were obtained for the sensor images were between 1 and 0.961 and for the phosphor plate images, the values ranged from 1 to 0.903 .

In relation to the general distribution of the errors (Table I), the most frequent sensor error was the absence of a safety margin $(35.7 \%)$. When the phosphor plate was used, the most prevalent error was an overlapping of the proximal surfaces of the teeth $(23.1 \%)$. The image of a cone cutting was not observed with the phosphor plate and no elongated images or long axis image errors were found for either of the two systems.

Table 1: General distribution of the types of errors observed in digital periapical radiographs performed with sensor and phosphor plate.

\begin{tabular}{|c|c|c|c|c|}
\hline \multirow{2}{*}{ Errors } & \multicolumn{2}{|c|}{ Sensor } & \multicolumn{2}{c|}{ Phosphor Plate } \\
\cline { 2 - 5 } & General & Frequency & General & Frequency \\
\cline { 2 - 5 } & $\mathbf{n}$ & $\mathbf{( \% )}$ & $\mathbf{n}$ & $\mathbf{( \% )}$ \\
\hline Elongated Image & - & - & - & - \\
\hline Shortened Image & 13 & 3.1 & 6 & 1.4 \\
\hline X-Ray Not Parallel to the Proximal surfaces of the Teeth & 112 & 26.7 & 97 & 23.1 \\
\hline Cone Cutting & 2 & 0.5 & - & - \\
\hline Absence of Safety Margin & 150 & 35.7 & 53 & 12.6 \\
\hline Excessive Safety Margin & 51 & 12.1 & 2 & 0.5 \\
\hline Non-Parallel Safety Margin & 43 & 10.2 & 61 & 14.5 \\
\hline Poor Receptor Centring & 78 & 18.6 & 55 & 13.1 \\
\hline Incorrect Long Axis of the Receptor & - & - & - & - \\
\hline Identification Mark Improperly Positioned & - & - & 38 & 9 \\
\hline
\end{tabular}


The distribution of the frequency of errors, according to the radiographed regions, is shown in Tables II and III. In the images that were obtained with the sensor (Table II), the most prevalent error was the absence of a safety margin, which was observed in all of the regions of the maxilla and mandibular. The upper and the lower molar regions showed the highest values, with both at $63.3 \%$. This was while the lowest values for the same error were in the upper right canine and in the lower left canine regions, both at $3.3 \%$. The image error with a cone cutting presented the smallest percentage error with the sensor, occurring only in the upper canine and in the lower molars, both on the right side $(3.3 \%)$. When the phosphor plate was used (Table III), the most frequent error was an overlapping of the proximal teeth surfaces, with a higher value for the lower premolar region on the left side $(66.7 \%)$.
This error was not observed in the upper and in the lower incisors, the right and the left lower canines and in the upper right premolars.

In relation to the frequency distribution of errors in the regions when using the sensor (Table II), the highest prevalence was in the lower right premolars. In this region, the greatest number of errors occurred with an overlapping of the proximal surfaces of the teeth $(70 \%)$, followed by the absence of a safety margin (56.7\%). While for the phosphor plate (Table III), the region with the highest prevalence was that of the lower right premolars, with a higher number of errors occurring for an overlapping of the proximal surfaces of the teeth $(63.3 \%)$, followed by the absence of a safety margin, together with them not being parallel, both at $43.3 \%$.

Table 2: Distribution of the frequency of errors, according to the regions, observed in periapical radiographs performed with sensor.

\begin{tabular}{|c|c|c|c|c|c|c|c|c|}
\hline \multirow{2}{*}{ Regions } & $\begin{array}{c}\text { Shortened } \\
\text { Image }\end{array}$ & $\begin{array}{c}\text { X-Ray Not- } \\
\text { Parallel to the } \\
\text { Proximal Surfaces }\end{array}$ & $\begin{array}{c}\text { Cone } \\
\text { Cutting }\end{array}$ & $\begin{array}{c}\text { Absence of } \\
\text { Safety Margin }\end{array}$ & $\begin{array}{c}\text { Excessive Safety } \\
\text { Margin }\end{array}$ & $\begin{array}{c}\text { Non-Parallel } \\
\text { Safety Margins }\end{array}$ & $\begin{array}{c}\text { Poor Receptor } \\
\text { Centring }\end{array}$ & $\begin{array}{c}\text { Incorrect Long Axis } \\
\text { of the Receptor }\end{array}$ \\
\cline { 2 - 9 } & $\mathbf{n}(\boldsymbol{\%})$ & $\mathbf{n}(\%)$ & $\mathbf{n}(\boldsymbol{\%})$ & $\mathbf{n}(\boldsymbol{\%})$ & $\mathbf{n}(\boldsymbol{\%})$ & $\mathbf{n}(\boldsymbol{\%})$ & $\mathbf{n}(\boldsymbol{\%})$ & $\mathbf{n}(\%)$ \\
\hline UI & $1(3.3)$ & - & - & $9(30)$ & - & $1(3.3)$ & $1(3.3)$ & - \\
\hline LI & - & - & - & $3(10)$ & - & $1(3.3)$ & $6(20)$ & - \\
\hline RUC & $2(6.7)$ & $15(50)$ & $1(3.3)$ & $1(3.3)$ & $7(23.3)$ & - & - & - \\
\hline LUC & $5(16.7)$ & $19(63.3)$ & - & $3(10)$ & $2(6.7)$ & - & $1(3.3)$ & - \\
\hline RLC & - & - & - & $6(20 \%)$ & $2(6.7)$ & - & $1(3.3)$ & - \\
\hline LLC & - & - & - & $1(3.3)$ & - & - & $2(6.7)$ & - \\
\hline RUPM & - & $6(20)$ & - & $16(53.3)$ & $7(23.3)$ & $9(30)$ & $1(3.3)$ & - \\
\hline LLPM & $1(3.3)$ & $12(40)$ & - & $14(46.7)$ & $6(20)$ & $9(30)$ & $1(3.3)$ & - \\
\hline RLPM & $2(6.7)$ & $21(70)$ & - & $17(56.7)$ & $6(20)$ & $7(23.3)$ & $14(46.7)$ & - \\
\hline LLPM & - & $18(60)$ & - & $13(43.3)$ & $5(16.7)$ & $9(30)$ & $2(6.7)$ & - \\
\hline RUM & - & $5(16.7)$ & - & $19(63.3)$ & $1(3.3)$ & $1(3.3)$ & $2(6.7)$ & - \\
\hline LUM & - & $3(10)$ & - & $19(63.3)$ & $4(13.3)$ & - & $5(16.7)$ & - \\
\hline LLM & $1(3.3)$ & $6(20)$ & $1(3.3)$ & $13(43.3)$ & $8(26.7)$ & $1(3.3)$ & $19(63.3)$ & - \\
\hline LLM & $1(3.3)$ & $7(23.3)$ & - & $16(53.3)$ & $3(10)$ & $5(16.7)$ & $23(76.7)$ & - \\
\hline
\end{tabular}

UI: Upper Incisors LLC: Left Lower Canine RUM: Right Upper Molars

LI: Lower Incisors RUPM: Right Upper Premolars LUM: Left Upper Molars

RUC: Right Upper Canine LLPM: Left Lower Premolars LLM: Right Lower Molars

LUC: Left Upper Canine RLPM: Right Lower Premolars LLM: Left Lower Molars

RLC: Right Lower Canine LLPM: Left Lower Premolars

Table 3: Distribution of the frequency of errors, according to the regions, observed in periapical radiographs performed with phosphor plate.

\begin{tabular}{|c|c|c|c|c|c|c|c|c|c|}
\hline Regions & $\begin{array}{l}\text { Shorte } \\
\text { ned } \\
\text { Image }\end{array}$ & $\begin{array}{c}\text { X-Ray Not- Parallel } \\
\text { to the Proximal } \\
\text { Surfaces }\end{array}$ & $\begin{array}{c}\text { Cone } \\
\text { Cutting }\end{array}$ & $\begin{array}{c}\text { Absence } \\
\text { of Safety } \\
\text { Margin } \\
\end{array}$ & $\begin{array}{l}\text { Excessive } \\
\text { Safety } \\
\text { Margin } \\
\end{array}$ & $\begin{array}{c}\text { Non-Parallel } \\
\text { Safety Margins }\end{array}$ & $\begin{array}{c}\text { Poor } \\
\text { Receptor } \\
\text { Centring }\end{array}$ & \begin{tabular}{|c|} 
Incorrect \\
Long Axis \\
of the Receptor
\end{tabular} & $\begin{array}{c}\text { Identification Mark } \\
\text { Improperly } \\
\text { Positioned } \\
\end{array}$ \\
\hline & n (\%) & n (\%) & n (\%) & n (\%) & n (\%) & n (\%) & n (\%) & n(\%) & \\
\hline UI & - & - & - & - & - & $1(3.3)$ & - & - & $2(6.7)$ \\
\hline LI & $1(3.3)$ & - & - & - & - & - & - & - & $3(10)$ \\
\hline RUC & - & $15(50)$ & - & - & - & - & - & - & $1(3.3)$ \\
\hline LUC & $1(3.3)$ & $11(36.7)$ & - & - & - & - & - & - & $1(3.3)$ \\
\hline RLC & - & - & - & $4(13.3)$ & - & - & $1(3.3)$ & - & \\
\hline LLC & $2(6.7)$ & - & - & - & - & - & - & - & $2(6.7)$ \\
\hline \begin{tabular}{|l|} 
RUPM \\
\end{tabular} & - & - & - & $4(13.3)$ & - & $16(53.3)$ & - & - & $1(3.3)$ \\
\hline LLPM & $1(3.3)$ & $15(50)$ & - & - & - & $13(43.3)$ & - & - & $2(6.7)$ \\
\hline \begin{tabular}{|l|} 
RLPM \\
\end{tabular} & - & $19(63.3)$ & - & $13(43.3)$ & $1(3.3)$ & $13(43.3)$ & $1(3.3)$ & - & $4(13.3)$ \\
\hline LLPM & - & $20(66.7)$ & - & $12(40)$ & - & $15(50)$ & - & - & $3(10)$ \\
\hline \begin{tabular}{|l|} 
RUM \\
\end{tabular} & - & $7(23.3)$ & - & $6(20)$ & - & - & $8(26.7)$ & - & $4(13.3)$ \\
\hline LUM & $1(3.3)$ & $5(16.7)$ & - & $6(20)$ & - & $1(3.3)$ & $3(10)$ & - & $2(6.7)$ \\
\hline LLM & - & $3(10)$ & - & $6(20)$ & $1(3.3)$ & $2(6.7)$ & $22(73.3)$ & - & $7(23.3)$ \\
\hline LLM & - & $2(6.7)$ & - & $2(6.7)$ & - & - & $2066.7)$ & - & $4(13.3)$ \\
\hline
\end{tabular}

UI: Upper Incisors LLC: Left Lower Canine RUM: Right Upper Molars

LI: Lower Incisors RUPM: Right Upper Premolars LUM: Left Upper Molars

RUC: Right Upper Canine LLPM: Left Lower Premolars LLM: Right Lower Molars

LUC: Left Upper Canine RLPM: Right Lower Premolars LLM: Left Lower Molars

RLC: Right Lower Canine LLPM: Left Lower Premolars 
When the two types of digital systems were compared in relation to the X-ray repetitions, a smaller number of errors was observed with the use of the phosphor plate, in which $7.7 \%$ of the radiographs were repeated (32), with a maximum of two repetitions for four radiographs. Whereas, with the sensor, 162 images needed to be repeated (38.5\%) and of these, four were repeated nine times (Table IV).

Table 4: Distribution of repetition frequency of digital periapical radiographs performed with sensor and phosphor plate.

\begin{tabular}{|c|c|c|c|c|}
\hline \multirow{2}{*}{ Repetitions } & \multicolumn{2}{|c|}{ Sensor } & \multicolumn{2}{c|}{ Phosphor Plate } \\
\cline { 2 - 5 } & General & Frequency & General & Frequency \\
\cline { 2 - 5 } & Radiographs & $\mathbf{( \% )}$ & Radiographs & $(\%)$ \\
\hline 0 & 258 & 61.4 & 388 & 92.4 \\
\hline 1 & 76 & 18.1 & 28 & 6.7 \\
\hline 2 & 38 & 9 & 4 & - \\
\hline 3 & 18 & 4.3 & - & - \\
\hline 4 & 6 & 1.4 & - & - \\
\hline 5 & 8 & 1.9 & - & - \\
\hline 6 & 2 & 0.5 & - & - \\
\hline 7 & 7 & 1,7 & - & - \\
\hline 8 & 3 & 0.7 & - & - \\
\hline 9 & 4 & 1 & & - \\
\hline
\end{tabular}

In a comparison between the two digital systems, the McNemar test showed statistically significant differences for the error of a poor receptor centering $(p=0.008)$, an excessive margin of safety $(\mathrm{p}=0.000)$ and the absence of a safety margin $(\mathrm{p}=0.000)$. The Kappa test, on the other hand, showed a high concordance for the elongated image, the cone cutting and an incorrect long axis of the receptor (Table V).

Table 5: Comparison between digital radiographic systems with phosphor plate and with sensor in the occurrence of technique errors in periapical radiographs

\begin{tabular}{|c|c|c|c|c|}
\hline \multirow{2}{*}{ ERRORS } & Plate & Sensor & McNemar & Kappa \\
\cline { 2 - 5 } & $\mathbf{n}$ & $\mathbf{n}$ & & \\
\hline Elongated Image & - & - & - & 1.000 \\
\hline Shortened Image & 6 & 13 & 0.167 & 0.020 \\
\hline X-Ray Not Parallel to the Proximal Surfaces & 97 & 112 & 0.151 & 0.396 \\
\hline Cone Cutting & - & 2 & - & 1.000 \\
\hline Absence of Safety Margin & 53 & 150 & 0.000 & 0.146 \\
\hline Excessive Safety Margin & 2 & 51 & 0.000 & 0.009 \\
\hline Non-Parallel Safety Margin & 61 & 43 & 0.057 & 0.126 \\
\hline Poor Receptor Centring & 55 & 78 & 0.008 & 0.387 \\
\hline$p<0.05$ Incorrect Long Axis of the Receptor & - & - & - & 1.000 \\
\hline
\end{tabular}

In the analyses of the results on the questionnaire that was answered by the students regarding the use of the digital systems, it was observed that $77.8 \%$ reported that they had already performed radiographs with a sensor. The advantages that were most cited for this system, when compared with the conventional technique, were the speed of an image acquisition $(50 \%)$; an elimination of the conventional image processing $(50 \%)$; the possibility of manipulating the image $(44.5 \%)$; less of a clinical time for the patient's care $(27.8 \%)$; and an elimination of the radiographic films and preserving the environment $(22.3 \%)$; the lower X-ray exposure for the patient was also cited, with a better quality in the radiography and in the digital image storage. Regarding the difficulties in the handling of the sensor, $77.8 \%$ answered that they had experienced difficulties; these included its rigidity, because it was uncomfortable for the patient (44.5\%); its thickness $(38.9 \%)$; as well as the necessary use of a positioner $(16.7 \%)$ and the sensor's cable (11\%).

In relation to the phosphor plate, $22.3 \%$ of the students had already performed X-rays with this type of an image receptor. The most reported advantages for this receptor, when compared with the conventional technique, were a fast image scanning, the phosphor plate's reuse and the image manipulation on the monitor, all with $22.3 \%$. The possibility of scanning several plates at the same time and the speed of the technique were also reported, both with $16.7 \%$. Regarding the difficulties in using the phosphor plate, $22.3 \%$ of the students described difficulties with this system; among them, the waiting time to reuse the plate (because the image must be erased for the new exposure); the use of a positioner; the time needed to scan the images and a lack of experience with the system, all with $5.5 \%$.

In a comparison between the two digital systems, $61 \%$ of the students preferred the sensor, $16.6 \%$ preferred the phosphor plate and $22.2 \%$ liked to use both systems. If the students could acquire one of the two digital radiographic systems, $83.4 \%$ of the students would buy the sensor, with the justifications of: (i) a reduction in the clinical time $(33.4 \%)$; (ii) the practicality of use $(22.2 \%)$; and (iii) obtaining an instantaneous image $(16.7 \%)$. While those who chose the phosphor plate were $16.6 \%$, with the justifications of: (i) the greater ease of handling the plate in the patient's mouth in the different regions $(16.7 \%)$; (ii) it was more comfortable for the patient (11\%); and (iii) it was a fast and easy image scanning procedure $(5.5 \%)$.

\section{Discussion}

In the present research, when comparing the two digital systems, those that had used the sensor presented the greatest number of errors in the periapical radiographic technique. The 
rigidity and the thickness of the sensor could justify this result, since they make the positioning in the mouth more difficult ${ }^{[2]}$. In contrast, the phosphor plate is less bulky than the sensor. It is similar in size, shape and thickness to the radiographic films and it can be used with the same positioner that is used in the conventional method ${ }^{[2,3]}$, unlike the sensor, which has a specific positioner. Another factor that may have hampered the realization of the technique with the sensor was the presence of the cable that makes the connection to the computer ${ }^{[4]}$. Thus, the similarity of the phosphor plate to the radiographic films may have contributed to the results obtained. This was quite evident, since the students participating in this research had had previous training, in order to perform the radiographic technique with films, while the system with a sensor was made available later, in all of the clinics of the Dentistry course.

In relation to the types of errors observed, the sensor achieved the most frequent errors, as follows: there was no safety margin $(35.7 \%)$ and there was an overlapping of the proximal surfaces of the teeth $(23.1 \%)$. For Senior et al. ${ }^{[2]}$, the most common error in the periapical radiographs, which caused a repetition of the images, was an absence of the apical region when using the sensor (37\%) and less, when using the phosphor plate $(6 \%)$. In the present study, the highest percentage of errors in the periapical radiographs also occurred with the use of the sensor. Several studies have compared the prevalence of errors when obtaining the images with the use of radiographic film and a sensor. For Sommers et al. ${ }^{[5]}$, the most common error with the sensor was an incorrect vertical angulation (53\%), while for Verteeget et al. [6], it was an incorrect horizontal positioning of the receiver and the crown cut. In the current research, the highest prevalence of errors in the periapical radiographic technique when using the sensor was no safety margin (35.7\%). Thus, when comparing the results of this current study, it was observed that the most common type of error with the sensor was not the same among the cited authors, but for both of the systems, the highest frequency of errors occurred when the sensor system was used.

Likewise, the comparisons between the phosphor plate and radiographic film were also verified. In a study performed by Tax et al. ${ }^{[7]}$, in the analyses of their images, they identified more errors of an overlap of the proximal surfaces (an incorrect horizontal angulation) with the use of the phosphor plate $(n=139)$ than with the film $(n=83)$, with no vertical errors in the images. For Zhang et al. ${ }^{[8]}$, the use of the phosphor plate also resulted in more overlapping errors of the proximal surfaces, while for an elongated image, a shortened image and a fold, these were the least found errors. These results have corroborated with those found in the current study, in which the highest percentage of errors with the use of the phosphor plate was for an overlapping of the proximal surfaces $(23.1 \%)$. For an elongated imaging, there were no errors and for a shortened imaging, the prevalence was small (1.4\%), being similar to the results of the above authors.

The frequency of errors, according to the X-ray region, for both the sensor and for the phosphor plate, was higher in the lower right premolars, with a higher prevalence for the proximal surface overlapping errors, $70 \%$ and $63.3 \%$, respectively. For Tax et al. ${ }^{[7]}$, the highest percentage of errors was also for the overlapping of the proximal surfaces, as well as being in the premolar region, with $59 \%$ occurring with the use of the phosphor plate. For Verteeget et al. ${ }^{[6]}$, the highest prevalence of errors was an incorrect horizontal positioning of the sensor and the crown cut, occurring more frequently in the molars $(17 \%)$ and in the anterior region $(28 \%)$. These results that have been obtained from the occurrence of more errors in the lower right premolars, may be due to the fact that this region is more atresic and together with the dental crowding in the mannequin, this may have hampered the positioning of the receptors. Senior et al. ${ }^{[2]}$ stated that the bulky sensors can be a challenge for an intraoral positioning, especially when the patients have a reduced mouth space.

In this current study, the dental teachers also analyzed the radiographic repetitions and there was a smaller number of repetitions with the phosphor plate. In a study by Verteeget $e t$ al. ${ }^{[6]}$, more radiographic repetitions were observed with the use of the sensor $(28 \%)$ than with the radiographic film $(6 \%)$. In another study, a total of 429 images were repeated, showing a higher percentage for the sensor $(77 \%)$ than for the phosphor plate $(23 \%)^{[2]}$. In both of these studies, the authors justified the higher prevalence of repetitions when using the sensor, by saying that it was possibly due to the obtainment of an instantaneous image and that this may have induced the dentists to re-expose more frequently. The results of the present study may also have to be carefully considered and rationalized, when regarding the high levels of repetition when using the sensor, a fact acknowledged and reported by the students themselves during their acquisition of the radiographs. Furthermore, the dose of radiation can be reduced on average by $60 \%$ to $65 \%{ }^{[9]}$, although there was an excessive number of repetitions in these digital systems. For Yosof et al. ${ }^{[10]}$ even if the exposure from dental X-rays is considered insignificant, it is not best practice to perform multiple radiographic examinations on the same person. These authors also considered that the students had problems in performing digital radiography in terms of choosing the right holder device, or deciding whether to use the holder or not and difficulty in properly placing the sensor inside the patient's mouth. It is also necessary that students have a better understanding of oral cavity morphology in order to correctly position the digital sensor. In addition, a greater emphasis on the study of oral anatomy is recommended ${ }^{[10]}$ and the teaching plan should incorporate the protocols of digital radiographs in the dental school ${ }^{[10,11]}$.

In the questionnaire that was answered by the students about the two digital systems used, several advantages and difficulties were reported, when compared to the radiographic film. The main advantage of the sensor that was cited was the speed of the image acquisition. This observation was also referred to by Senior et al. ${ }^{[2]}$, who showed the benefits of using a sensor, for the instantaneous display of the images and the absence of any conventional processing. Another advantage to be reported when using the sensor was the protection of the environment. Several studies have shown the benefits of such digital systems, by an elimination of the darkroom, the films themselves and the wastage of processing equipment, such as the processing chemicals and the lead foil $[4,12]$. This was in an agreement with the responses of the students. The image storage was also mentioned in the questionnaire, since the images were easily archived in a digital medium and transferred electronically, without any impairment in image quality ${ }^{[4]}$.

The possibility of scanning multiple phosphor plates at the same time was one of the advantages reported by the students, because there are systems available in which the scanning of several plates can be performed in a single step, while for the other systems, the scanning is performed individually ${ }^{[13]}$. For both of these two digital systems, the manipulation of the images was pointed out as one of the advantages. The 
radiographs, when obtained by these digital systems, can be manipulated by using the tools of the programs and thus, it improves the images and the information, in order to aid in the diagnosis ${ }^{[10]}$. The disadvantages as described by the students when using the sensor were: the cable was connected to the computer and its rigidity and its thickness; these disadvantages have also been mentioned in other studies ${ }^{[4,15]}$. The images that were obtained with the phosphor plate should be desensitized before being reused ${ }^{[16]}$; this being one of the disadvantages cited by the students, since it implies a waiting time for their reuse. In addition, the time for the phosphor plate scanning was seen as a difficulty. This was also reported in the study by Wenzel et al. ${ }^{[3]}$, showing that the working time differed between the sensors and the phosphor plates.

In the preferences of the students for the two digital systems, $61 \%$ chose the sensor, $16 \%$ the phosphor plate and $22 \%$ liked to work with both systems. Sommers et al. ${ }^{[5]}$ performed a study on the periapical radiographic technique, by using a sensor and radiographic film; although $74 \%$ of the students had difficulties in positioning the sensor on the mannequin, they also reported as having preferred to use the sensor for the rapid acquisition of the images. Digital radiographic systems facilitate the acquisition and the analysis of radiographs, aiding in a better diagnosis. However, it was observed that the students still have difficulties when performing the X-rays with these two systems, especially with the sensor. Therefore, further training should be conducted, in order to avoid the repetition of the radiographs, as well as to the non-exposure of patients to unnecessary doses of radiation.

\section{Conclusions}

According to the results that were obtained in this study, it was possible to conclude that:

- There were a greater number of errors and repetitions with the use of the sensor in the periapical radiographic technique;

- For both of the systems, the most occurred errors were in the region of the lower right premolars.

\section{References}

1. White SC, Pharoah MJ. The evolution and application of the dental maxillofacial imaging modalities. Dent Clin North Am. 2008; 52(4):689-705.

2. Senior A, Winand C, Ganatra S, Lai H, Alsulfyani N, Pereira CP. Digital intraoral imaging re-exposure rates of dental students. J Dent Educ. 2018; 82(1):61-68.

3. Wenzel A, Møystad A. Work flow with digital intraoral radiography: A systematic review. Acta Odontol Scandinavia. 2010; 68(2):106-114.

4. White SC, Pharoah MJ. Oral Radiology: Principles and Interpretation. 7th ed. St. Louis: Mosby Inc, 2014.

5. Sommers TM, Mauriello SM, Ludlow JB, Platin E, Tyndall DA. Pre-clinical performance comparing intraoral film and CCDbased systems. J Dent Hyg. 2002; 76(1):26-33.

6. Versteeg CH, Sanderink GC, van Ginkel FC, PF. An evaluation of periapical radiography with a chargecoupled device. Dentomaxillofac Radiol. 1998; 27(2):97101.

7. Tax CL, Robb CL, Brillant MG, Doucette HJ. Integrating Photo-Stimulable Phosphor plates into dental and dental hygiene radiography curricula. J Dent Educ. 2013; 77(11):1451-1460.

8. Zhang W, Huynh CP, Abramovitch K, Leon IL, Arvizu L. Comparison of technique errors of intraoral radiographs taken on film $\mathrm{v}$ photostimulable phosphor (PSP) plates. Tex Dent J. 2012; 129(6):589-96.

9. Duarte HEM, Araújo CCM, Sannomiya EK. [Digital systems in dento-maxillofacial radiology: a new phase in dentistry?] [Article in Portuguese] Rev Bras Odontol. 2008; 65(1):42-47.

10. Yusof MYPM, Nur Liyana Rahman A, Asri AAA, Othman NI, Mokhtar IW. Repeat analysis of intraoral digital imaging performed by undergraduate students using a complementary metal oxide semiconductor sensor: An institutional case study. Imaging Sci. Dent. 2017; 47:233-9.

11. Gopal SK, Krishnaraj N, Priya M. Faulty radiographs: A retrospective radiographic analysis. Int. J Appl. Dent Sci. 2018; 4(1):72-76.

12. Wenzel A. Computer-aided image manipulation of intraoral radiographs to enhance diagnosis in dental practice: a review. Int Dent J. 1993; 43(2):99-108.

13. Van Der Stelt PF. Filmless imaging: The uses of digital radiography in dental practice. J Am Dent Assoc. 2005; 136(10):1379-1387.

14. Candeiro GTM, Bringel ASF, Vale IS. [Digital Radiology: Literature review] [Article in Portuguese] Rev Odontol Araçatuba. 2009; 30(2):38-44.

15. Paurazas SB, Geist JR, Pink FE. Comparison of diagnostic accuracy of digital imaging using CCD and CMOS-APS sensors with E-speed film in the detection of periapical bony lesions. Oral Surg Oral Med Oral Pathol Oral Radiol Endod. 2000; 89(3):356-362.

16. Neto FH, de Melo DP. [Digital radiography] [Article in Portuguese]. Rev ABRO. 2010; 11(1):5-17. 